\title{
Developing a questionnaire to measure patients' beliefs about inhaler treatment for asthma: Tests of validity and reliability
}

\author{
CH Hand, S Morley and M Adams
}

Christopher Hand

General Practitioner and Honorary Senior Lecturer

\section{Sylvia Morley}

Senior Research Associate

\section{Malcolm Adams}

Clinical Psychologist

School of Health Policy and Practice, University of East Anglia, Norwich NR4 7TJ, UK

Correspondence to: Dr CH Hand

c.hand@uea.ac.uk

Date received: 17/04/00

Date accepted: 03/05/00

Prim. Care Respir. J. 2000:9(1);12-15

\begin{abstract}
Aim: To develop a valid and reliable questionnaire for measuring patients' beliefs about inhaler treatments for asthma.

Methods: In seven general practices, 335 patients prescribed both beclomethasone and salbutamol inhalers completed a 66-item questionnaire. Subscales were developed using factor analysis and a 29-item questionnaire sent to 90 patients in another practice to estimate test-retest reliability.

Results: Three factors explained $31.4 \%$ of the variance relating to beliefs about inhalers: preventing asthma symptoms, problems with and concerns about inhalers, and relieving asthma symptoms. Cronbach's alpha coefficients of the sub-scales were $0.71,0.70$ and 0.70 respectively. Intra-class correlation coefficients were 0.87 or more for all three sub-scales. The prevention and relief scale scores were significantly correlated with estimates of corticosteroid and $\beta$-agonist use.

Conclusions: A valid and reliable questionnaire has been developed for measuring patients' beliefs about inhaler treatments for asthma. Its utility in predicting adherence and facilitating patient education needs to be tested.
\end{abstract}

\section{INTRODUCTION}

Poor adherence to corticosteroid inhaler treatment for the prevention of asthma is common, as is over-reliance on short-acting $\beta$-agonists. ${ }^{1}$ Qualitative research has revealed the importance of patients' beliefs about therapy in asthma and these beliefs are likely to be of importance in the way that patients use inhalers. ${ }^{2}$ Although psychological models have been used to explain and predict health behaviours, relatively little work has been done to develop instruments to measure beliefs and attitudes to asthma treatment. ${ }^{3-6}$ Such instruments could be used to identify those patients who hold beliefs that are inconsistent with the optimal use of asthma treatment. This information would be of value in discussions between patients and health professionals. ${ }^{7}$ The instrument could also be used as an outcome measure in studies investigating the effectiveness of educational programmes to improve adherence. Such a questionnaire is being developed

\section{Box 1: Summary of different types of validity}

- Face validity: the items in a test look as if they are measuring what they are supposed to measure and is acceptable to test user and subjects.

- Content validity: the ability of a measure to sample adequately the domain which it is measuring.

- Concurrent validity: the correlation of a new test with existing tests measuring the same concepts.

- Predictive validity: how well a measure predicts a particular criterion.

- Construct validity: how well a measure relates to various phenomena that would be predicted by theory.

- Convergent validity: how strongly different measures of the same construct relate to one another.

- Discriminant validity: the extent to which a measure has low correlations with measures of different constructs.

and this paper describes its further refinement, including an examination of the validity and reliability of its sub-scales. ${ }^{8-10}$

Other questionnaires have been developed to assess adherence to asthma treatments. Brooks et al devised a five-item scale that had moderate internal reliability with Cronbach's alpha coefficients of 0.67, 0.75 and 0.68 in their analyses. ${ }^{5}$ The scale, however, does not differentiate between inhalers used for prevention or relief. Six items in Creer and colleagues' 76-item Revised Asthma Behavior Checklist refer to medication use. ${ }^{11}$ The items do not refer to specific medications and their psychometric properties have not been explored separately. Apter et al developed a four-item attitude toward inhaled steroid scale, but this had poor internal reliability $(0.51) .{ }^{12}$ They also developed a more reliable $(0.74)$ patient-clinician sixitem communication scale that contained three items about inhalers. A questionnaire has to be valid as well as reliable; the definitions of the different types of validity are summarised in Box 1 . There is often a reciprocal relationship between validity and reliability. This paper illustrates the practical application of some of these theoretical principles in the development of a questionnaire.

\section{METHODS}

Early development of the questionnaire

The items were originally identified from semistructured interviews with eight patients with asthma from one practice in Suffolk. The interviews were analysed qualitatively and eight main themes identified. Patients' views were allocated to each theme, resulting in 102 items, 92 of which covered beliefs about inhaler treatment. Forty patients from the same practice were then interviewed using these items and their responses scored on a five-point Likert scale. The responses were correlated with estimates of beclomethasone and salbutamol use. ${ }^{8}$ Factor analysis of those beliefs that had the highest correlations with inhaler use was then performed and 33 items selected. ${ }^{9}$ A 66-item questionnaire was completed by 335 asthma patients from seven general practices in Norfolk. They rated the 33 items on a five-point scale first in relation to beclomethasone and then in relation to salbutamol. ${ }^{10}$

Development of the 29-item questionnaire For the factor analysis several items were dropped using the following criteria. Four items with the highest number of missing responses (from six to eight) were excluded. Frequency distributions of all the remaining responses were examined and those items that had less than $10 \%$ of their responses on one side and more than $75 \%$ on the other side of the fivepoint scale were excluded. Eleven items were lost using this criterion. An item analysis was performed and four further items were dropped as their individual correlations with the total item score were statistically insignificant. ${ }^{13}$ Principal components analysis was 
then performed, with pairwise selection of missing variables, and Varimax rotation. Nine factors emerged with Eigenvalues greater than one, accounting for $60 \%$ of the variance. Examination of forced three, four and five factor solutions suggested that a three-factor solution provided the best description; balancing variance explained, interpretability and reliability. The factor analysis was repeated for patients aged under 50 and for practices with a response rate of over $60 \%$. This was done to examine the effects of excluding older patients who may have chronic obstructive airways disease and practices with low response rates that might have affected the generalisability of the results.

Exploring the Cronbach's alpha coefficients of the sub-scales, some items were reselected and others removed to produce three sub-scales of similar reliability. Four items loaded significantly on more than one factor. Although this is undesirable, it is, to some extent, inevitable when using factor analytic methods that impose orthogonal solutions - i.e. require factors to be independent. One approach would be to eliminate these items so that each factor is assessed by items that load onto one factor only. One item was dropped, but the other three were kept in as excluding them would have reduced the reliability of the resulting scales. Cronbach's alpha coefficients of the final prevention, relief, and concerns sub-scales were 0.71 , 0.70 and 0.70 respectively. The revised 29 -item questionnaire is in Table 1 and demonstrates the effect on Cronbach's alpha of dropping individual items.

\section{Test-retest reliability}

In a different Norfolk practice this questionnaire, with three additional questions relating to morbidity from asthma, was sent by post to all the patients between the ages of 16 and 60 prescribed a combination of beclomethasone and salbutamol. ${ }^{14}$ A letter explaining the study and a stamped addressed envelope were included. A second questionnaire was sent two weeks later. Reminders were not sent. Intra-class correlations were performed to explore test-retest reliability of the scales. ${ }^{15}$ Further checks were made on the internal reliability of the scales.

Sub-scales, inhaler use and morbidity

Estimates of inhaler use were made from the number
Cronbach's alpha with item removed

0.69

0.68

0.70

0.64

0.70

0.69

0.68

0.66

0.69

0.66

0.68

0.70

0.68

0.68

0.70

0.69

0.69

0.68

Not applicable

Not applicable

0.66

0.67

0.66

0.69

0.68

0.66

0.71

0.66

of inhalers ordered over the previous year on the computerised repeat prescription system. Spearman's rank correlation coefficients were performed between the sub-scale scores, inhaler use and morbidity.

\section{RESULTS}

\section{Development of the 29-item questionnaire}

Three factors explained $31.4 \%$ of the variance of the responses. The sub-scale items, their factor loadings and variance of each factor, are shown in Table 2. The factors can be interpreted as preventing asthma symptoms, problems with and concerns about inhalers, and relieving asthma symptoms. Analysis using only those patients aged under 50 or practices with a response rate of over $60 \%$ yielded almost identical results.

\section{Test-retest reliability}

Seventy-four patients $(82.2 \%)$ returned the first questionnaire and, of those patients, 58 returned the second questionnaire $(78.4 \%)$. Women $(36 ; 62.1 \%)$ were more likely to return both questionnaires than men $(22 ; 37.9 \%)$, this difference approaching 
Table 2: Beliefs relating to inhaler use, with factor loadings of greater than 0.32 , percentage of variance explained by each factor and Eigenvalues. Two items relating to personal control with low loadings on the third factor are shown in brackets.

Beliefs

Factor loadings

$12-3$

Factor 1. Preventing asthma symptoms; variance explained 12.4\%; Eigenvalue 3.5

I find having my preventer by me is reassuring

I think my preventer inhaler keeps me feeling well

0.68

I prefer to collect my preventer inhalers on a regular basis

0.65

I usually plan ahead to get my preventer inhalers

The preventer inhaler is effective at helping me when I have asthma

0.60

The preventer inhaler has more of a local effect on my lungs than

a general effect on my body

How I manage my preventer inhaler is a shared decision

between my doctor and me

Preventing an asthma attack with my reliever inhale

is better than waiting for one to happen

I understand how my preventer inhaler works on my asthma

0.47

0.35

Factor 2. Problems and concerns about inhalers; variance explained 10.5\%; Eigenvalue 2.9

I am worried about possible long-term side effects from my reliever inhaler

I am worried about possible long-term side effects from my preventer inhaler

0.73

0.69

should like more information about the way the preventer inhaler works on

my lungs and the effects that it has on me

There is never enough time to talk to my doctor about how I feel about

my reliever inhaler

I should like to stop my reliever inhaler and see how I am without it

I feel embarrassed using a reliever inhaler in public

It would be helpful to have something to make taking my reliever inhaler easier

I don't like being dependent on my reliever inhaler

I find it difficult to know when my reliever inhaler is running out

Taking my reliever inhaler is inconvenient

Factor 3. Relieving asthma symptoms; variance explained 8.5\%; Eigenvalue 2.4

I think that I should take my reliever inhaler regularly

I really rely on my reliever inhaler

I prefer my reliever inhaler to my preventer inhaler

I usually take my reliever and preventer inhalers together, one after the other

I like to have several reliever inhalers and put them in different places

I would prefer to take a tablet than use a preventer inhaler

(Avoiding the things that trigger my asthma is sometimes more effective in controlling my asthma than using a preventer inhaler)

(Relaxing and keeping calm is sometimes more effective in controlling my asthma than using my reliever inhaler)

Sub-scales, inhaler use and morbidity

Spearman rank correlations between the sub-scale scores, inhaler use and morbidity are shown in Table 3. Those patients using less than two puffs per day of either inhaler had lower scores on the prevention (Mann-Whitney $\mathrm{U}=68.5 ; p=0.02)$ and relief sub-scales (Mann-Whitney $\mathrm{U}=164.5$; $p=0.038$ ) that were statistically significant compared to those using more than two puffs per day. Those patients apparently not using corticosteroid inhalers had lower scores on the concerns sub-scale that were also statistically significant (Mann-Whitney $\mathrm{U}=42.5$; $p=0.044$ ).

\section{DISCUSSION}

The process of developing this questionnaire has been a long and careful one. The items are grounded upon the views of patients with asthma and these views also relate to their use of inhalers. Rand and Wise advocated the development of a standardised, validated and reliable self-report questionnaire that could be applied across different research settings and this measure goes a long way towards meeting that need. ${ }^{6}$ Patients and health professionals have found the questionnaire both acceptable and comprehensive. This confirms that the sub-scales have both face and content validity. The prevention and relief sub-scales correlate with estimated use of inhaled corticosteroids and short-acting $\beta$-agonists demonstrating construct validity. Construct validity is also

statistical significance $\left(\chi^{2}=3.823 ; p=0.076\right)$. The median age of responders was 41 (interquartile range 31-52) and those who were older were also more likely to return both questionnaires (Mann-Whitney $\mathrm{U}=633 ; p=0.03)$.

Test-retest reliability using intra-class coefficients was 0.87 for the whole questionnaire and $0.88,0.87$ and 0.91 for the prevention, relief, and problems and concerns sub-scales respectively. Cronbach's alpha coefficients remained at satisfactory levels in two of the sub-scales $(0.77,0.68,0.75)$, showing acceptable internal reliability in a cross-validation sample. One patient's pair of questionnaires was excluded from this analysis as both questionnaires were returned on the same day and the responses identical. Morbidity scores did not change significantly. confirmed by the fact that the relief sub-scale correlates positively with the Jones morbidity index. This correlation is in the theoretically expected direction as the index has been shown to correlate with $\beta$-agonist use. ${ }^{14}$ The negative correlation with the prevention sub-scale is understandable given that beliefs promoting the use of preventive treatment should reduce morbidity. Convergent validity is demonstrated by the positive correlation between the prevention and relief sub-scales. This is also to be expected given that most patients are taking both inhalers and many of them are taking them at the same time. The difference between the relief and prevention sub-scales in their correlations with morbidity shows evidence of discriminant validity. In addition, discriminant validity is confirmed by the fact that those patients using less than two puffs per day of 
either inhaler had lower scores on the prevention and relief subscales compared to those using more than two puffs per day. Perhaps paradoxically, those patients who had not collected a prescription for a corticosteroid inhaler in the previous year had significantly lower scores on the concerns sub-scale. In those patients who had, there was a positive, but not statistically significant, correlation between the number of puffs of corticosteroid used and a higher score on this subscale. This would suggest that those patients apparently not taking corticosteroids are not concerned about side effects, whilst those taking them may well be and as the dose increases so does the concern. There does not appear to be the same problem with $\beta$ agonists. This hypothesis needs further testing.The internal reliabilities of the sub-scales are acceptable, although the reliever scale did not perform quite as well as expected in the second phase of the study. It is possible to inflate internal reliability by adding items that do not necessarily improve the content validity of a scale. The test-retest reliabilities of the questionnaire and sub-scales are very high although the interval between the two tests is relatively short.

There are two main limitations to this study. ${ }^{10}$ Patients who are not taking their inhalers, or taking them infrequently, are under-represented and patients from surgeries with low response rates may not be representative of the asthma population as a whole. In the test-retest part of the study, which had a much higher response, there was a big variation in the number of inhalers ordered by those who returned questionnaires. The responses to those items that were common to both questionnaires were almost identical (data available from the authors). In addition, excluding patients from practices with low response rates did not materially affect the result of the factor analysis. This suggests that different patterns of inhaler use are probably represented in both surveys.

Two items relating to personal control had low loadings on the third (relief) factor. A fourth sub-scale may need to be developed. This would be in keeping with the concepts of self-management with inhaler treatment and patients' views about managing asthma without inhalers. In the next phase of testing the predictive and concurrent validity of the questionnaire, these two items will be compared with the control items in the Illness Perception Questionnaire. ${ }^{16}$

\section{CONCLUSION}

A valid and reliable questionnaire has been developed for measuring patients' beliefs about inhaler treatments for asthma. Its utility in predicting adherence and facilitating patient education needs to be tested. The questionnaire may also have a use as an outcome measure both in clinical and research settings.

\section{References}

1. Hand CH. Adherence and asthma. In: Myers L, Midence K, editors. Adherence in medical conditions. London: Harwood Academic Press; 1998

2. Adams S, Pill R, Jones A. Medication, chronic illness and identity: The perspective of people with asthma. Soc Sci Med 1997;45:189-201

3. Conner M, Norman P. Predicting health behaviour. Buckingham: Open University Press; 1996

4. Richards JM, Bailey WC, Windsor RA et al. Some simple scales for use in asthma research. J Asthma 1988;25:363-71

5. Brooks CM, Richards JM, Kohler CL et al. Assessing adherence to asthma medication and inhaler regimens: A psychometric analysis of adult self-report scales. Med Care 1994;32:298-307

6. Rand CS, Wise RA. Measuring adherence to asthma medication regimens. Am J Respir Crit Care 1994;149:S59-S76

7. Royal Pharmaceutical Society of Great Britain. From compliance to concordance: Towards shared goals in medicine taking. London: RPS; 1997

8. Hand $\mathrm{CH}$, Bradley C. Health beliefs of adults with asthma: Toward an understanding of the difference between symptomatic and preventive use of inhaler treatment. J Asthma 1996;33:331-8

9. Hand $\mathrm{CH}$. Developing a questionnaire to measure patients beliefs about inhaler treatment: A pilot study. Asthma Gen Pract 1998;6:40-3

10. Hand $\mathrm{CH}$, Morley S, Adams M. Patients' beliefs about inhalers: Are they compatible with the optimal use of inhalers for the prevention and relief of asthma? Asthma Gen Pract 2000;8(1):9-11

11. Creer TL, Wigal JK, Tobin DL et al. The revised asthma problem behavior checklist. J Asthma 1989;26:17-29

12. Apter AJ, Reisine ST, Affleck G et al. Adherence to twicedaily dosing of inhaled steroids. Am J Respir Crit Care Med 1998;157:1810-7

13. Kline P. An easy guide to factor analysis. London: Routledge; 1994

14. Jones K, Cleary R, Hyland M. Predictive value of a simple asthma morbidity index in a general practice population. $\mathrm{Br}$ J Gen Pract 1999;49:23-6

15. Everitt B, Hay D. Talking about statistics. New York: Wiley; 1992

16. Weinman J, Petrie KJ, Moss-Morris R et al. The Illness Perception Questionnaire: A new method for assessing cognitive representations of illness. Psychol Health 1996;11:431-45 